
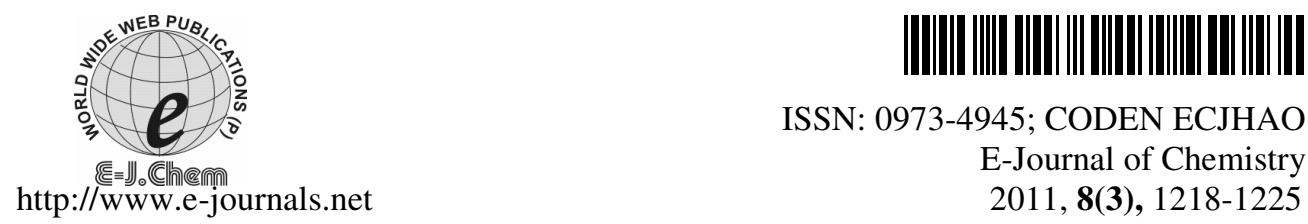

ISSN: 0973-4945; CODEN ECJHAO

E-Journal of Chemistry

2011, 8(3), 1218-1225

\title{
Assessment of Dyeing Properties of Novel Bisazo- Bisazomethine Disperse Dyes on Polyester Fabric
}

\author{
B. C. DIXIT ${ }^{*}$ and D. M. PATEL \\ Department of Chemistry \\ V. P. \& R. P. T. P. Science College, Vallabh Vidyanagar-388120 \\ Dist. Anand, State-Gujarat, India \\ dixits20002003@yahoo.co.in
}

Received 21 September 2010; Revised 19 December 2010; Accepted 8 January 2011

\begin{abstract}
Novel bisazo-bisazomethine disperse dyes were prepared by the coupling of diazotized solutions of various aromatic amines with 2,2'-\{ sulfonylbis [4,1-phenylene nitrilomethylylidene]\} diphenol (SB). Above Schiff base was prepared by the condensation of 2-hydroxybenzaldehyde with 4,4'-sulphonyldianiline (Dapson). The resultant dyes were characterized by elemental analysis, IR and ${ }^{1} \mathrm{H}$ NMR spectral studies. The UV Visible absorption spectral data were investigated in dimethylformamide (DMF) and are discussed in terms of structural property relationship. Their dyeing performance as disperse dyes has been assessed on polyester fabrics. The results show that a better hue was obtained on polyester fabrics and have mild to moderate fastness properties.
\end{abstract}

Keywords: Dapson, Schiff base, Bisazo-bisazomethine disperse dyes, Polyester fabrics, Chromophoric group

\section{Introduction}

Since long back azo disperse dyes, azo-vat and azo-acid dyes etc. were marketed as a commercial dyes. All have phenolic moiety bearing hydroxy group(s) as an auxochrome ${ }^{1-5}$. Much attention has also been given towards bisazo and polyazo dyes as dying materials ${ }^{6-11}$. In addition to that there are several reports regarding bisazomethine dyes in which azoimine group formed by Schiff's reaction of aldehyde with aromatic diamine ${ }^{12-14}$. However, there are no any reports regarding bisazo-bisazomethine disperse dyes in which both bisazo and bisazomethine chromophoric groups lying in a single molecular framework. Hence, the present communication comprises synthesis; characterization and dyeing performance of bisazo-bisazomethine disperse dyes on polyester fabric. 


\section{Experimental}

All the chemicals used were of analytical grade and were further purified as and when required. Dapson (4,4'-sulfonyldianiline), salicylaldehyde (2-hydroxybenzaldehyde), different aromatic primary amines, sodium hydroxide, sodium nitrite, hydrochloric acid were purchased from local market. The organic solvents used were purified by standard methods ${ }^{15}$. The aromatic amines used for diazotization are listed in Scheme 1. Polyester fabrics were gifted by Color Tax (Pvt) Ltd, Surat. Melting points were determined by open capillary method and were found uncorrected. The visible absorption spectra were measured on a Carl Zeiss UV/VIS specord spectrometer and elemental analysis was carried out on Perkin Elmer CHNS/O Analyzer 2400 Series II. Infrared spectra were recorded in $\mathrm{KBr}$ pellets on a Perkin-Elmer spectrum GX FT-IR model. ${ }^{1} \mathrm{H}$ NMR spectra were recorded on Hitachi R-1500 in DMSO-D 6 solvent and TLC (Thin layer chromatography) was run on a aluminium sheets precoated with silica gel $60 \mathrm{~F}_{245}$, (Merck, Germany) using ethyl acetate: $n$-hexane (1:1) solvent system. Colour spot was visualized by UV chamber. Intra dyeing machine was used for dying purpose.

\section{Synthesis of 2,2'-\{sulfonylbis[4,1-phenylenenitrilomethylylidene]\}diphenol (SB)}

A solution of dapson $(4.96 \mathrm{~g}, 0.02 \mathrm{~mol})$ in absolute alcohol $(50 \mathrm{~mL})$ was prepared. To the above solution an ethanolic solution $(100 \mathrm{~mL})$ of salicylaldehyde $(4.88 \mathrm{~g}, 0.04 \mathrm{~mol})$ was added slowly under stirring to produce a yellow crystalline solid product upon cooling at room temperature. The product thus obtained was filtered, washed with ethanol and dried in vacuum desiccator. Yield: $79.65 \%$ m.p. $221-224{ }^{0} \mathrm{C}$ (uncorrected). The synthetic route is shown in Scheme 1.

\section{Synthesis of the bisazo-bisazomethine disperse dyes}

\section{Diazotization procedure}

Bisazo-bisazomethine disperse dyes were prepared in a two steps viz. diazotization of aromatic amines followed by their coupling with Schiff base. Diazotization was carried out by the reported method ${ }^{16-18}$. Accordingly, aniline and other substituted amines such as $p$-toludine, 2-nitroaniline, 3-nitroaniline, 4-nitroaniline, 3-chloroaniline, 4-chloroanilne, 4-bromoaniline, 3-aminophenol and 4-aminophenol were diazotized by following the given procedure:

A solution of $1.86 \mathrm{~g}$ aniline $(0.02$ mole $)$ in $25 \mathrm{~mL} 2 \mathrm{~N} \mathrm{HCI}(0.05$ mole $)$ was cooled in an ice bath and was stirred well. After reaching to $0-5{ }^{\circ} \mathrm{C}$ temperature a chilled $10 \mathrm{~mL}$ solution of $1.38 \mathrm{~g}$ sodium nitrite $(0.02$ mole) was added drop wise so that the temperature should not exceeds $2-3{ }^{0} \mathrm{C}$ during a time of half an hour. The mixture was stirred well further for about $10 \mathrm{~min}$. after completion of diazotization reaction. The test conducted for this purpose is a blue colouration with Congo red paper and a weak blue colour with starch iodide paper. If diazotization is not completed than add more nitrite solution drop wise until a positive test for diazotization is obtained. The diazotized amine solution thus prepared was used immediately for coupling reaction. Almost all amines were diazotized by following the similar procedure.

Coupling of above mentioned diazotized aromatic amines with Schiff base was carried out by following the reported method ${ }^{16-18}$. Accordingly, $4.56 \mathrm{~g}$ Schiff base $(0.01 \mathrm{~mole})$ was dissolved in aqueous solution of $50 \mathrm{~mL}$ sodium hydroxide $(0.01 \mathrm{~mole})$. The clear solution was cooled in ice bath and diazonium salt solution of amine ( 0.02 mole) was added drop by drop over a period of 15 minutes accompanied by vigorous stirring. The $\mathrm{pH}$ of solution 
mixture was maintained in the range of 8 to 9 by simultaneous addition of $10 \%$ w/v sodium carbonate solution. Stirring was continued for $2 \mathrm{~h}$, allowing the temperature to rise to ambient. Then $2 \mathrm{M}$ solution of acetic acid was added until the $\mathrm{pH}$ of solution becomes 7 . The dye precipitated out was then filtered off, washed with distilled water until it becomes salts free and was dried at room temperature. The dyes were recrystallised by appropriate organic solvent and were designated as dyes $\mathrm{D}_{1}-\mathrm{D}_{10}$.

\section{Dyeing and fastness properties of fabrics}

All the dyes $D_{1}$ to $D_{10}$ were applied on polyester fabrics in $2 \%$ shade by using the reported procedure $^{19}$. The fastness to light, sublimation and perspiration of the dye pattern was assessed according to British standard 1006-1978 and the washing fastness according to Indian standard (ISO method) at $60{ }^{\circ} \mathrm{C} / 30 \mathrm{~min}$. The rubbing fastness was tested using crock meter (10 stroke To \& fro) (Atlas) AATCC-1961. The percentage of exhaustion and fixation of dyes on polyester fabric was determined by following the reported method ${ }^{20}$.

\section{Results and Discussion}

All the dyes were obtained as amorphous powder ranging in colour from yellowish orange to dark brown. The TLC results showed that only a single spot was observed for each dye.

\section{Characteristic data of Schiff's base and dyes}

2,2'-\{Sulfonylbis[4,1-phenylenenitrilomethylylidene] $\}$ diphenol (SB). Yield: 79.65\%, m.p.: 221-223 ${ }^{\circ} \mathrm{C}$ (uncorrected). Calculated for $\mathrm{C}_{26} \mathrm{H}_{20} \mathrm{~N}_{2} \mathrm{O}_{4} \mathrm{~S}$ : m.wt.: 456; C, $68.42 \% ; \mathrm{H}, 4.38 \%$; $\mathrm{N}, 6.41 \%$; S $7.01 \%$. Found: C, 68.20\%; H, 4.18\%; N, 6.31\%; S, 6.95\%. IR $\left(\mathrm{KBr}, \mathrm{cm}^{-1}\right)$ : $3032(\mathrm{C}-\mathrm{H}$ aromatic), $1650(>\mathrm{C}=\mathrm{N}-), 1495(\mathrm{C}=\mathrm{C}$ aromatic), $1325(\mathrm{C}-\mathrm{N}), 1195,1375$ (phenol, $\mathrm{O}-\mathrm{H}$ bending and $\mathrm{O}-\mathrm{C}$ stretching), 1145, 1305 (sulfones $-\mathrm{SO}_{2}-$ ). ${ }^{1} \mathrm{H}-\mathrm{NMR}(400$ MHz, DMSO-D 6 solvent, $\delta / \mathrm{ppm}), 6.8-8.17(16 \mathrm{H}, \mathrm{m}, \mathrm{Ar}-\mathrm{H}), 8.52(2 \mathrm{H}, \mathrm{s}, \mathrm{N}=\mathrm{CH}), 12.63$ $(2 \mathrm{H}, \mathrm{s}, \mathrm{Ar}-\mathrm{OH})$.

\section{2-[(4-\{4-[(2-Hydroxy-5-p-tolylazobenzylidene)amino]benzenesulfonyl $\}$ phenylimino $)$} methyl]-4-p-tolylazo phenol $\left(\boldsymbol{D}_{1}\right)$

Yield: $64.16 \%$, m.p.: $135-137{ }^{0} \mathrm{C}$ (uncorrected). $\mathrm{R}_{\mathrm{f}}$ value: 0.88 ; Calculated for $\mathrm{C}_{40} \mathrm{H}_{32} \mathrm{~N}_{6} \mathrm{O}_{4} \mathrm{~S}$ : m.wt.: 692; C, 69.36\%; H, 4.62\%; N, 12.13\%; S, 4.62\%. Found: C, 69.25\%; H, 4.33\%; N, $12.04 \%$; S, 4.50\%. IR ( $\left.\mathrm{KBr}, \mathrm{cm}^{-1}\right) 3036(\mathrm{C}-\mathrm{H}$ aromatic), 1631 (>C=N-), $1590(-\mathrm{N}=\mathrm{N}-)$, $1435\left(-\mathrm{CH}_{3} \mathrm{C}-\mathrm{H}\right.$ bending), 1180, 1336 (phenol, $\mathrm{O}-\mathrm{H}$ bending and $\mathrm{O}-\mathrm{C}$ stretching), $1340\left(\mathrm{C}-\mathrm{N}\right.$ ), 1499, 1456 (C-C multiple aromatic), 1445, 1278 (sulfones $-\mathrm{SO}_{2}-$ ), 825 (aromatic substitution, two adjacent $\mathrm{H}$-atom). ${ }^{1} \mathrm{H}-\mathrm{NMR}\left(400 \mathrm{MHz}, \mathrm{DMSO}-\mathrm{D}_{6}\right.$ solvent, $\delta$ / ppm) $2.24\left(6 \mathrm{H}, \mathrm{s}, \mathrm{Ar}-\mathrm{CH}_{3}\right), 6.88-8.17(22 \mathrm{H}, \mathrm{m}, \mathrm{Ar}-\mathrm{H}), 8.46(2 \mathrm{H}, \mathrm{s}, \mathrm{N}=\mathrm{CH}), 12.46$ $(2 \mathrm{H}, \mathrm{s}, \mathrm{Ar}-\mathrm{OH})$.

\section{2-[(4-\{4-[(2-Hydroxy-5-phenlazobenzylidene)amino]benzenesulfonyl\}phenylimino) methyl]-4-phenylazophenol $\left(\boldsymbol{D}_{2}\right)$}

Yield: $71.08 \%$, m.p.: $141-143{ }^{\circ} \mathrm{C}$ (uncorrected), $\mathrm{R}_{\mathrm{f}}$ value: 0.87 . Calculated for $\mathrm{C}_{38} \mathrm{H}_{28} \mathrm{~N}_{6} \mathrm{O}_{4} \mathrm{~S}$ : m.wt.: 664; C, 66.67\%; H, 4.21\%; N, 12.65\%; S, 4.81\%. Found: C, 68.45\%; H, 4.11\%; N, $12.5 \% ; \mathrm{S}, 4.75 \%$. IR $\left(\mathrm{KBr}, \mathrm{cm}^{-1}\right): 3029(\mathrm{C}-\mathrm{H}$ aromatic), $1632(>\mathrm{C}=\mathrm{N}-), 1594(-\mathrm{N}=\mathrm{N}-)$, 1181, 1332 (phenol, O-H bending and O-C stretching), $1339(\mathrm{C}-\mathrm{N}), 1499,1456(\mathrm{C}-\mathrm{C}$ multiple aromatic), 1150, 1290 (sulfones $-\mathrm{SO}_{2}-$ ), 832 (aromatic substitution, two adjacent $\mathrm{H}$-atom). ${ }^{1} \mathrm{H}-\mathrm{NMR}\left(400 \mathrm{MHz}, \mathrm{DMSO}-\mathrm{D}_{6}\right.$ solvent, $\delta$ / ppm) 7.28-8.16 (24H, m, Ar-H), 8.45 $(2 \mathrm{H}, \mathrm{s}, \mathrm{N}=\mathrm{CH}), 12.44(2 \mathrm{H}, \mathrm{s}, \mathrm{Ar}-\mathrm{OH})$. 
2-\{[4-(4-\{[2-Hydroxy-5-(4-nitrophenylazo)benzylidene\}aminobenzenesulfonyl) phenylimino] methyl\}-4-(4-nitrophenylazo)phenol $\left(\boldsymbol{D}_{3}\right)$

Yield: $65.30 \%$, m.p.: $156.158{ }^{0} \mathrm{C}$ (uncorrected), $\mathrm{R}_{\mathrm{f}}$ value: 0.86 . Calculated for $\mathrm{C}_{38} \mathrm{H}_{26} \mathrm{~N}_{8} \mathrm{O}_{8} \mathrm{~S}$ : m.wt.: 754; C, 60.47\%; H, 3.44\%; N, $14.85 \%$; S, 4.24\%. Found: C, 60.41\%; H, 3.40\%; N, $14.75 \%$; S, 4.20\%. IR ( $\left.\mathrm{KBr}, \mathrm{cm}^{-1}\right): 3241(\mathrm{C}-\mathrm{H}$ aromatic), $1663(>\mathrm{C}=\mathrm{N}-), 1589(-\mathrm{N}=\mathrm{N}-)$, 1502, $1334\left(\mathrm{C}-\mathrm{NO}_{2}\right.$ aromatic), 1186, 1378 (phenol, $\mathrm{O}-\mathrm{H}$ bending and $\mathrm{O}-\mathrm{C}$ stretching), $1388(\mathrm{C}-\mathrm{N}), 1635,1589,1435$ (C-C multiple aromatic) 1146, 1278 (sulfones $-\mathrm{SO}_{2}-$ ), 832 (aromatic substitution, two adjacent $\mathrm{H}$-atom). ${ }^{1} \mathrm{H}-\mathrm{NMR}\left(400 \mathrm{MHz}, \mathrm{DMSO}-\mathrm{D}_{6}\right.$ solvent, $\delta / \mathrm{ppm})$ 7.2-8.17 (22H, m, Ar-H), $8.47(2 \mathrm{H}, \mathrm{s}, \mathrm{N}=\mathrm{CH}), 12.47(2 \mathrm{H}, \mathrm{s}, \mathrm{Ar}-\mathrm{OH})$.

2-\{[4-(4-\{[2-Hydroxy-5-(3-nitrophenylazo)benzylidene]amino\}benzenesulfonyl) phenylimino]methyl-4-(2-nitrophenylazo)phenol $\left(\boldsymbol{D}_{4}\right)$

Yield: $58.35 \%$, m.p.: $137-139{ }^{0} \mathrm{C}$ (uncorrected), $\mathrm{R}_{\mathbf{f}}$ value: 0.85 . Calculated for $\mathrm{C}_{38} \mathrm{H}_{26} \mathrm{~N}_{8} \mathrm{O}_{8} \mathrm{~S}$ : m.wt.: 754; C, 60.47\%; H, 3.44\%; N, 14.85\%; S, 4.24\% Found: C, 60.35\%; H, 3.35\%; N, $14.78 \%$; S, 4.20\%. IR ( $\left.\mathrm{KBr}, \mathrm{cm}^{-1}\right): 3032(\mathrm{C}-\mathrm{H}$ aromatic), $1633(>\mathrm{C}=\mathrm{N}-), 1587(-\mathrm{N}=\mathrm{N}-)$, 1504, $1345\left(\mathrm{C}-\mathrm{NO}_{2}\right.$ aromatic), 1185, 1374 (phenol $\mathrm{O}-\mathrm{H}$ bending and $\mathrm{O}-\mathrm{C}$ stretching), 1336 $\left(\mathrm{C}-\mathrm{N}\right.$ ), 1633, 1585, 1433 (C-C multiple aromatic), 1145, 1305 (sulfones - $\mathrm{SO}_{2}-$ ), 832 (aromatic substitution two adjacent $\mathrm{H}$-atom). ${ }^{1} \mathrm{H}-\mathrm{NMR}(400 \mathrm{MHz}$, DMSO-D 6 solvent, $\delta / \mathrm{ppm})$ 6.90-8.47 (22H, m, Ar-H), $8.49(2 \mathrm{H}, \mathrm{s}, \mathrm{N}=\mathrm{CH}), 12.43(2 \mathrm{H}, \mathrm{s}, \mathrm{Ar}-\mathrm{OH})$.

4-(3-Chlorophenylazo)-2-\{[4(4-\{[5-(3-chlorophenylazo)-2-hydroxybenzylidene] amino\}benzenesulfonyl)phenyliminomethyl phenol $\left(\boldsymbol{D}_{5}\right)$

Yield: $75.58 \%$, m.p.: $148-150{ }^{0} \mathrm{C}$ (uncorrected), $\mathrm{R}_{\mathrm{f}}$ value: 0.94 . Calculated for $\mathrm{C}_{38} \mathrm{H}_{26} \mathrm{~N}_{6} \mathrm{Cl}_{2} \mathrm{O}_{4} \mathrm{~S}$ : m.wt.: 733; C,62.21\%; H, 3.54\%; N, $11.45 \% ; \mathrm{Cl}, 9.68 \% ; \mathrm{S}, 4.36 \%$ Found: $\mathrm{C}, 62.15 \% ; \mathrm{H}, 3.51 \% ; \mathrm{N}, 11.36 \% ; \mathrm{Cl}, 9.55 \% ; \mathrm{S}, 4.31 \%$. IR $\left(\mathrm{KBr}, \mathrm{cm}^{-1}\right): 3035(\mathrm{C}-\mathrm{H}$ aromatic), $1631(>\mathrm{C}=\mathrm{N}-), 1585(-\mathrm{N}=\mathrm{N}-), 1184,1377$ (phenol $\mathrm{O}-\mathrm{H}$ bending and $\mathrm{O}-\mathrm{C}$ stretching), 1601, 1584, 1498 (C-C multiple aromatic), $1335(\mathrm{C}-\mathrm{N}), 1150,1300$ (sulfones $\left.\mathrm{SO}_{2}-\right), 833$ (aromatic substitution two adjacent $\mathrm{H}$-atom), $710(\mathrm{C}-\mathrm{Cl}) .{ }^{1} \mathrm{H}-\mathrm{NMR}(400 \mathrm{MHz}$, DMSO-D $_{6}$ solvent, $\left.\delta / \mathrm{ppm}\right)$ 7.0-8.27 (22H, m, Ar-H), $8.44(2 \mathrm{H}, \mathrm{s}, \mathrm{N}=\mathrm{CH}), 12.47(2 \mathrm{H}, \mathrm{s}$, $\mathrm{Ar}-\mathrm{OH})$.

2-\{[4-(4-\{[2-Hydroxy-5-(2-nitrophenylazo)benzylidene]amino\}benzenesulfonyl) phenylimino methyl\}-4-(2-nitrophenylazo)phenol $\left(\boldsymbol{D}_{6}\right)$

Yield: $78.78 \%$, m.p.: $152-155{ }^{\circ} \mathrm{C}$ (uncorrected), $\mathrm{R}_{\mathrm{f}}$ value: 0.92 . Calculated for $\mathrm{C}_{38} \mathrm{H}_{26} \mathrm{~N}_{8} \mathrm{O}_{8} \mathrm{~S}$ : m.wt: 754; C, 60.47\%; H, 3.44\%; N, 14.85\%; S, 4.24\%. Found: C, 60.35\%; H, 3.40\%; N, 14.20\%; S, 4.14\%. IR (KBr, cm $\left.{ }^{-1}\right): 3030(\mathrm{C}-\mathrm{H}$ aromatic), $1640(>\mathrm{C}=\mathrm{N}-), 1590(-\mathrm{N}=\mathrm{N}-)$, 1510, $1350\left(\mathrm{C}-\mathrm{NO}_{2}\right.$ aromatic), 1183, 1370 (phenol, $\mathrm{O}-\mathrm{H}$ bending and $\mathrm{O}-\mathrm{C}$ stretching), 1605, 1575, 1507 (C-C multiple bond aromatic), $1334(\mathrm{C}-\mathrm{N}), 1148,1307$ (sulfones $-\mathrm{SO}_{2}-$ ), 833 (aromatic substitution, two adjacent $\mathrm{H}$-atom). ${ }^{1} \mathrm{H}-\mathrm{NMR}\left(400 \mathrm{MHz}, \mathrm{DMSO}^{-\mathrm{D}_{6}}\right.$ solvent, $\delta$ / ppm) 6.95-8.21 (22H, m, Ar-H), 8.45 (2H, s, N=CH), 12.49 (2H, s, Ar-OH).

4-(4-Chlorophenylazo)2-\{[4-(4-\{[5-(4-chlorophenylazo)-2-hydroxybenzylidene amino benzenesulfonyl)phenylimino]methyl phenol $\left(\boldsymbol{D}_{7}\right)$

Yield: $75.0 \%$, m.p.: $150-152{ }^{0} \mathrm{C}$ (uncorrected), $\mathrm{R}_{\mathrm{f}}$ value: 0.88 . Calculated for $\mathrm{C}_{38} \mathrm{H}_{26} \mathrm{~N}_{6} \mathrm{Cl}_{2} \mathrm{O}_{4} \mathrm{~S}$ : m.wt: 733; C, 62.21\%; H, 3.54\%; N, $11.45 \%$; S, 4.36\%; Cl, 9.68\%. Found: $\mathrm{C}, 62.11 \% ; \mathrm{H}, 3.45 \% ; \mathrm{N}, 11.40 \% ; \mathrm{S}, 4.26 \% ; 9.61 \%$. IR (KBr, $\left.\mathrm{cm}^{-1}\right): 3238$ (C-H aromatic), $1632(>\mathrm{C}=\mathrm{N}-), 1591(-\mathrm{N}=\mathrm{N}-), 1178,1397$. (phenol $\mathrm{O}-\mathrm{H}$ bending and $\mathrm{O}-\mathrm{C}$ stretching), 
1632, 1540, 1492 (C-C multiple bond aromatic), $1333(\mathrm{C}-\mathrm{N}), 1145,1279$ (sulfones $\left.\mathrm{SO}_{2}-\right), 694(\mathrm{C}-\mathrm{Cl}), 831$ (aromatic substitution, two adjacent $\mathrm{H}$-atom). ${ }^{1} \mathrm{H}-\mathrm{NMR}$ (400 MHz, DMSO-D 6 solvent, $\delta / \mathrm{ppm}) 7.19-8.15(22 \mathrm{H}, \mathrm{m}, \mathrm{Ar}-\mathrm{H}), 8.46(2 \mathrm{H}, \mathrm{s}, \mathrm{N}=\mathrm{CH})$, $12.48(2 \mathrm{H}, \mathrm{s}, \mathrm{Ar}-\mathrm{OH})$.

4-(4-Hydroxyphenylazo)-2-\{[4-(4-\{[5-(4-hydroxyphenylazo)-2-hydroxybenzylidene] amino benzenesulfonyl)phenylimino]methyl phenol $\left(\boldsymbol{D}_{8}\right)$

Yield: 55.56\%, m.p.: $139-141{ }^{0} \mathrm{C}$ (uncorrected), $\mathrm{R}_{\mathrm{f}}$ value: 0.85 . Calculated for $\mathrm{C}_{38} \mathrm{H}_{28} \mathrm{~N}_{6} \mathrm{O}_{6} \mathrm{~S}$ : m.wt.: 696; C, 65.51\%; H, 4.02\%; N, $12.06 \%$; S, 4.59\%. Found: C, $65.38 \%$; H, 3.95\%; N, $11.98 \%$; S, 4.45\%. IR ( $\left.\mathrm{KBr}, \mathrm{cm}^{-1}\right): 3032$ (C-H aromatic), $1631(>\mathrm{C}=\mathrm{N}-), 1595(-\mathrm{N}=\mathrm{N}-)$, 1195, 1399 (phenol O-H bending and O-C stretching), 1610, 1590, 1490 (C-C multiple bond aromatic), $1331(\mathrm{C}-\mathrm{N}), 1148,1280$ (sulfones $-\mathrm{SO}_{2}-$ ), 831 (aromatic substitution, two adjacent $\mathrm{H}$-atom). ${ }^{1} \mathrm{H}-\mathrm{NMR}\left(400 \mathrm{MHz}, \mathrm{DMSO}^{-\mathrm{D}_{6}}\right.$ solvent, $\left.\delta / \mathrm{ppm}\right) 6.87-8.18(22 \mathrm{H}, \mathrm{m}$, $\mathrm{Ar}-\mathrm{H}), 8.45$ (2H, s, N=CH), 12.44 (2H, s, Ar-OH terminal), 10.84 (2H, s, Ar-OH).

\section{4-(3-Hydroxyphenylazo)-2-\{[4-(4-\{[5-(3-hydroxyphenylazo)-2-hydroxybenzylidene]} amino benzenesulfonyl)phenylimino]methyl phenol $\left(\boldsymbol{D}_{\mathbf{9}}\right)$

Yield: $73.89 \%$, m.p.: $171-173{ }^{0} \mathrm{C}$ (uncorrected), $\mathrm{R}_{\mathrm{f}}$ value: 0.83 . Calculated for $\mathrm{C}_{38} \mathrm{H}_{28} \mathrm{~N}_{6} \mathrm{O}_{6} \mathrm{~S}$ : m.wt.: 696; C, 65.51\%; H, 4.02\%; N, 12.06\%; S, 4.59\%. Found: C, 65.35\%; H, 4.03\%; N, $12.02 \% ; \mathrm{S}, 4.49 \%$. IR $\left(\mathrm{KBr}, \mathrm{cm}^{-1}\right): 3063$ (C-H aromatic), $1625(>\mathrm{C}=\mathrm{N}-)$, $1591(-\mathrm{N}=\mathrm{N}-), 1187,1380$ (phenol $\mathrm{O}-\mathrm{H}$ bending and $\mathrm{O}-\mathrm{C}$ stretching), $1337(\mathrm{C}-\mathrm{N})$, $1624,1591,1500$ (C-C multiple bond aromatic), 1146, 1278 (sulfones $-\mathrm{SO}_{2}-$ ), 833 (aromatic substitution, two adjacent $\mathrm{H}$-atom). ${ }^{1} \mathrm{H}-\mathrm{NMR}\left(400 \mathrm{MHz}, \mathrm{DMSO}-\mathrm{D}_{6}\right.$ solvent, $\delta / \mathrm{ppm}) 6.88-8.13(22 \mathrm{H}, \mathrm{m}, \mathrm{Ar}-\mathrm{H}), 8.46(2 \mathrm{H}, \mathrm{s}, \mathrm{N}=\mathrm{CH}), 9.91(2 \mathrm{H}, \mathrm{s}, \mathrm{Ar}-\mathrm{OH}$ terminal), $12.48(2 \mathrm{H}, \mathrm{s}, \mathrm{Ar}-\mathrm{OH})$.

4-(4-Bromophenylazo)-2-\{[4-(4-\{[5-(4-bromophenylazo)-2-hydroxybenzylidene] amino $b$ benzenesulfonyl)phenylimino]methyl phenol $\left(\boldsymbol{D}_{10}\right)$

Yield: $57.18 \%$, m.p.: $155-157{ }^{0} \mathrm{C}$ (uncorrected), $\mathrm{R}_{\mathrm{f}}$ value: 0.88 . Calculated for $\mathrm{C}_{38} \mathrm{H}_{26} \mathrm{~N}_{6} \mathrm{Br}_{2} \mathrm{O}_{4} \mathrm{~S}$ : m.wt.: 822; C, 55.47\%; H, 3.16\%; N, $10.21 \%$; Br, $19.46 \% ; \mathrm{S}, 3.89 \%$. Found: C, 55.37\%; H, 3.11\%; N, 10.15\%; Br, 19.35\%; S, 3.75\%. IR (KBr, $\left.\mathrm{cm}^{-1}\right): 3080$ (C-H aromatic), $1633(>\mathrm{C}=\mathrm{N}-), 1585(-\mathrm{N}=\mathrm{N}-), 1180,1375$. (phenol $\mathrm{O}-\mathrm{H}$ bending and O-C stretching), 1340 (C-N), 1595, 1570, 1495 (C-C multiple bond aromatic), 1144, 1310 (sulfones $-\mathrm{SO}_{2}-$ ), $1340(\mathrm{C}-\mathrm{N}), 835$ (aromatic substitution, two adjacent $\mathrm{H}$-atom), 545 (C-Br). ${ }^{1} \mathrm{H}-\mathrm{NMR}\left(400 \mathrm{MHz}, \mathrm{DMSO}_{6} \mathrm{D}_{6}\right.$ solvent, $\delta$ / ppm) 7.18-8.16 (22H, m, Ar-H), 8.44 $(2 \mathrm{H}, \mathrm{s}, \mathrm{N}=\mathrm{CH}), 12.42$ (2H, s, Ar-OH).

The result of the elemental analysis of each dye is consistent with the predicted structure as shown in Scheme 1. The number of azo groups and methine groups are two in each dye molecule. The nitrogen content and number of azo groups and number of azomethine groups are correlated with the expected values. IR spectrum of each dye exhibited the important features of aromatic, azo, hydroxyl, azomethine, nitro, halo and sulfonyl groups. The ${ }^{1} \mathrm{H}$ NMR spectra also showed the important signals at their expected positions for all bisazo-bisazomethine disperse dyes. Thus, the structure of bisazo-bisazomethine disperse dyes were confirmed as shown in Scheme 1. The absorption maxima $\left(\lambda_{\max }\right)$ in the visible spectra of all dyes recorded in DMF fall in the range of 420-535 $\mathrm{nm}$ as shown in Table 1. The value of the logarithm of the molar extinction coefficient $(\log \varepsilon)$ of all the dyes were in the range of 4.15-4.41, which consistent with their high absorption intensity. 

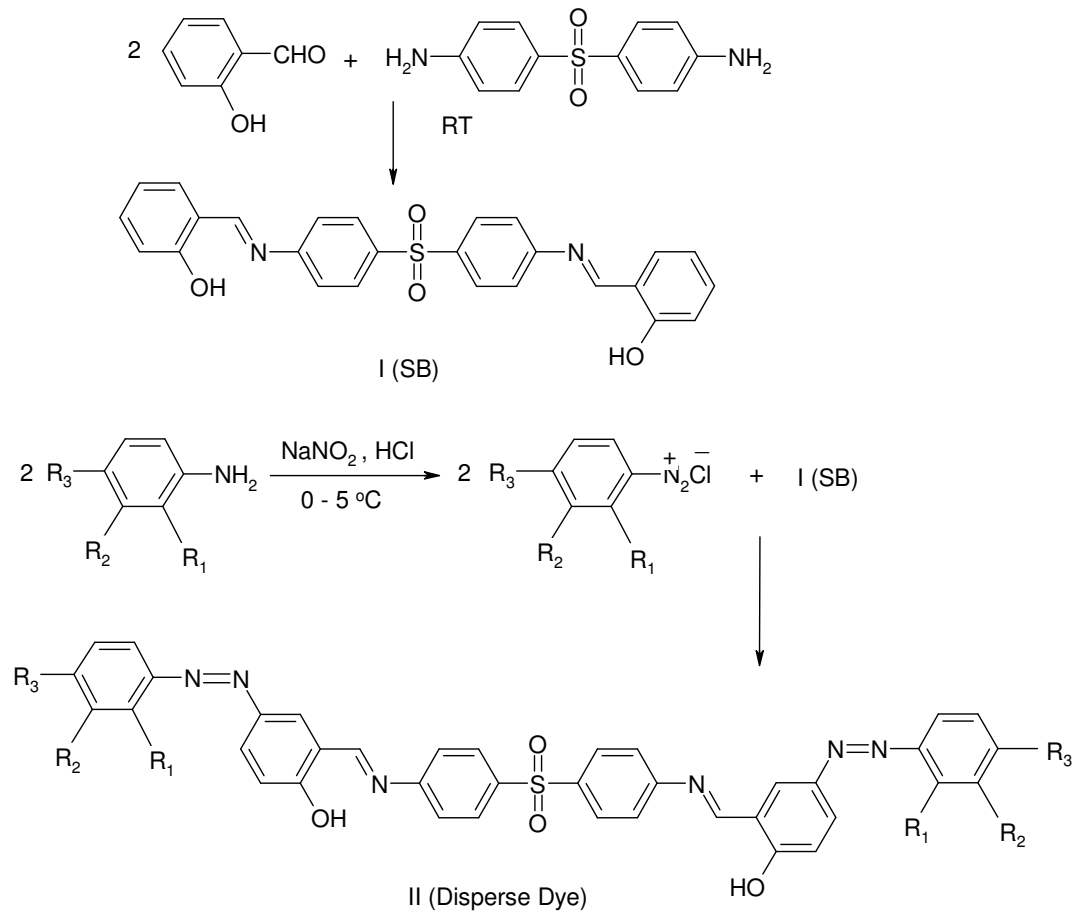

Scheme 1. Synthetic route for the preparation of bisazo-bisazomethine disperse dyes $\left\{D_{1}-D_{10}\right\}$ Where $R_{1}, R_{2}$ and $R_{3}$ substituent of aromatic amines are given below:

\begin{tabular}{ccccc}
\hline Dye No. & $\mathrm{R}_{1}$ & $\mathrm{R}_{2}$ & $\mathrm{R}_{3}$ & Amines \\
\hline $\mathrm{D}_{1}$ & $\mathrm{H}$ & $\mathrm{H}$ & $\mathrm{CH}_{3}$ & 4-Methylaniline \\
$\mathrm{D}_{2}$ & $\mathrm{H}$ & $\mathrm{H}$ & $\mathrm{H}$ & Aminobenzene \\
$\mathrm{D}_{3}$ & $\mathrm{H}$ & $\mathrm{H}$ & $\mathrm{NO}_{2}$ & 4-Nitroaniline \\
$\mathrm{D}_{4}$ & $\mathrm{H}$ & $\mathrm{NO}_{2}$ & $\mathrm{H}$ & 3-Nitroaniline \\
$\mathrm{D}_{5}$ & $\mathrm{H}$ & $\mathrm{Cl}$ & $\mathrm{H}$ & 3-Chloroaniline \\
$\mathrm{D}_{6}$ & $\mathrm{NO}_{2}$ & $\mathrm{H}$ & $\mathrm{H}$ & 2-Nitroaniline \\
$\mathrm{D}_{7}$ & $\mathrm{H}$ & $\mathrm{H}$ & $\mathrm{Cl}$ & 4-Chloroaniline \\
$\mathrm{D}_{8}$ & $\mathrm{H}$ & $\mathrm{H}$ & $\mathrm{OH}$ & 4-Hydroxyaniline \\
$\mathrm{D}_{9}$ & $\mathrm{H}$ & $\mathrm{H}$ & $\mathrm{H}$ & 3-Hydroxyaniline \\
$\mathrm{D}_{10}$ & $\mathrm{H}$ & $\mathrm{H}$ & $\mathrm{Br}$ & 4-Bromoaniline \\
\hline
\end{tabular}

Moreover, the presence of electron donating or electron attracting groups did not bring about any marked increase in $\lambda_{\max }$ in the visible region and $\log \varepsilon$ remained nearly constant. However, electron attracting substitutions such as $-\mathrm{Cl}$ and $-\mathrm{NO}_{2}$ increase the polarizability. This leads to a decrease in the energy between the highest occupied molecular orbital and lowest unoccupied molecular orbital and thus, the $\pi \rightarrow \pi^{*}$ electronic transition occurs with lower frequency photons, resulting in a bathochromic shift of the visible absorption band.

\section{Dying properties of the dyes}

The dispersed dyes were applied at $2 \%$ depth on polyester fabric. The result of exhaustion of the dye bath and the fixation of the dyed fabric are given in Table 1 . These dyes gave a wide range of colours varying from yellowish orange to dark brown with good levelness, brightness and depth on the fabric. The variation in the shades of the dye fabric results from 
both the nature and position of the substituent present on the diazotised compound. The dyeing showed an excellent fastness to washing, perspiration and sublimation but showed poor rubbing fastness and is shown in Table 2. A remarkable degree of levelness after washing was observed. This may be attributed to the good penetration into and affinity of the dye for the fabric structure.

Table 1. Absorption maxima $\left(\lambda_{\max }\right)$, intensities $(\log \varepsilon)$, exhaustion $(E)$ and fixation $(F)$ of bisazo-bisazomethine disperse dyes on polyester fabric

\begin{tabular}{cccccc}
\hline Dye & \multicolumn{2}{c}{$\begin{array}{c}\text { Absorption maxima } \\
\text { No. }\end{array}$} & & \multicolumn{2}{c}{ Disperse dyeing on polyester } \\
\cline { 4 - 5 } & $\lambda_{\max } / \mathrm{nm}$ in DMF & $\log \varepsilon$ & $\% \mathrm{E}$ & $\% \mathrm{~F}$ \\
\hline $\mathrm{D}_{1}$ & 440 & 4.33 & 74 & 77 \\
$\mathrm{D}_{2}$ & 420 & 4.15 & 67 & 78 \\
$\mathrm{D}_{3}$ & 535 & 4.41 & 65 & 66 \\
$\mathrm{D}_{4}$ & 472 & 4.22 & 68 & 71 \\
$\mathrm{D}_{5}$ & 441 & 4.32 & 72 & 71 \\
$\mathrm{D}_{6}$ & 481 & 4.41 & 70 & 68 \\
$\mathrm{D}_{7}$ & 460 & 4.32 & 79 & 76 \\
$\mathrm{D}_{8}$ & 490 & 4.26 & 75 & 69 \\
$\mathrm{D}_{9}$ & 433 & 4.28 & 82 & 82 \\
$\mathrm{D}_{10}$ & 428 & 4.17 & 62 & 63 \\
\hline
\end{tabular}

Table 2. Results of bisazo-bisazomethine disperse dyeing and various fastness properties of the dye on polyester fabric

\begin{tabular}{|c|c|c|c|c|c|c|c|c|}
\hline \multirow{2}{*}{$\begin{array}{l}\text { Dye } \\
\text { no. }\end{array}$} & \multirow{2}{*}{$\begin{array}{l}\text { Color shades on } \\
\text { polyester fabric }\end{array}$} & \multirow{2}{*}{$\begin{array}{l}\text { Light } \\
\text { fastness }\end{array}$} & \multirow{2}{*}{$\begin{array}{l}\text { Washing } \\
\text { fastness }\end{array}$} & \multicolumn{2}{|c|}{$\begin{array}{l}\text { Perspiration } \\
\text { fastness }\end{array}$} & \multirow{2}{*}{$\begin{array}{l}\text { Sublimation } \\
\text { fastness }\end{array}$} & \multicolumn{2}{|c|}{$\begin{array}{l}\text { Rubbing } \\
\text { fastness }\end{array}$} \\
\hline & & & & Acid & Alkaline & & Dry & Wet \\
\hline $\mathrm{D}_{1}$ & Yellowish orange & 5 & 5 & 4.5 & 5 & 5 & 3 & 3 \\
\hline $\mathrm{D}_{2}$ & Light Orange & 5 & 4 & 5 & 5 & 5 & 3.4 & 3.4 \\
\hline $\mathrm{D}_{3}$ & Light Brown & 5 & 4 & 5 & 5 & 5 & 3 & 3 \\
\hline $\mathrm{D}_{4}$ & Brownish Yellow & 4.4 & 5 & 4.5 & 5 & 5 & 4 & 3.4 \\
\hline $\mathrm{D}_{5}$ & Light Orange & 5 & 5 & 5 & 5 & 4 & 3.3 & 3.4 \\
\hline $\mathrm{D}_{6}$ & Yellowish Brown & 4.5 & 5 & 5 & 5 & 5 & 4 & 3.4 \\
\hline $\mathrm{D}_{7}$ & Light Orange & 4 & 4.5 & 4 & 4.5 & 4 & 3.3 & 3.3 \\
\hline $\mathrm{D}_{8}$ & Dark Yellow & 4 & 3 & 4 & 4 & 5 & 3 & 3 \\
\hline $\mathrm{D}_{9}$ & Dark Yellow & 5 & 4.5 & 5 & 5 & 5 & 3.4 & 3 \\
\hline $\mathrm{D}_{10}$ & Dark Brown & 5 & 5 & 5.3 & 5 & 5 & 3 & 3.4 \\
\hline
\end{tabular}

\section{Conclusion}

Novel bisazo-bisazomethine disperse dyes were prepared and duly characterized. They have good fastness to light, sublimation and perspiration properties but exhibit poor rubbing fastness. The nature of the substituent in the coupling components has little influence on the visible absorption and the shade of the dyeing.

\section{Acknowledgment}

The authors are grateful to the principal, for providing the necessary research facilities. 


\section{References}

1. $\quad$ Mohamed S K and Nour EI-Din A M, J Chem Res., 1999, 8, 508-509.

2. Peters A T and Walker D, J Chem Soc., 1956, 1429-1436

3. Naik R D, Desai C K and Desai K R, Orient J Chem., 2000, 16, 159.

4. Gorden P F and Gregory P, Organic Chemistry in Color, $1^{\text {st }}$ Ed., Springer Verlag, Berlin, Heidelberg, New York, 1983, 60.

5. Yurteri S, Onen A and Yagci Y, Eur Polym J., 2002, 38, 1845-1850.

6. Griffiths J, Bello K A and Chong L, J Chem Soc., Perkin Trasactions, 1981, 2, 815.

7. Stopa B, Konieczny L, Piekarska B, Roteman I, Rybarska J and Skowronek M, Biochimie., 1997, 79, 23-26.

8. Dayananda B P and Revanasiddappa H D, Chemical Papers, 2007, 61(6), 446-451.

9. Wang M, Funabiki K and Matsui M, Dyes and Pigments, 2003, 57(1), 77-86.

10. Mali N J, Mistry B D and Desai K R, Oriental J Chem., 2000, 16, 185.

11. Matsui M, Kamino Y, Hayashi M, Funabiki K, Shibata K, Muramatsu H, Abe Y and Kaneko M, Liq Cryst., 1998, 25, 235-240.

12. Asiri A M, Dyes and Pigments, 1999, 42, 209.

13. Asiri A M, Bull Korean Chem Soc., 2003, 24(4), 426-430.

14. Asiri A M, J Chem Soc Pak., 2004, 26, 57-60.

15. Vogel A I, A Textbook of Practical Organic Chemistry, $3^{\text {rd }}$ Ed., Longman, London 1961, 620.

16. Frirz-David H E and Blengy L, Fundamental process of Dye Chemistry, $3^{\text {rd }}$ Ed., Wiley, New York, 1949, 241.

17. Szymczyk M, Shafei A E and Freeman H S, Dyes and Pigments, 2007, 72, 8.

18. Dixit B C, Patel H M and Desai D J, J Serb Chem Soc., 2007, 72(2), 119.

19. Maradiya H R and Patel V S, High Perform Polym., 2000, 12, 335.

20. Maradiya H R and Patel V S, Fibres and Polym., 2002, 3, 43. 


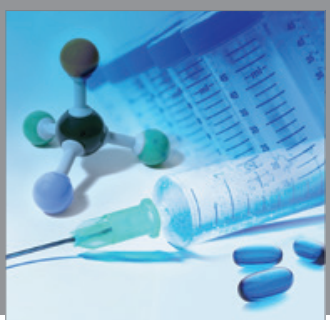

International Journal of

Medicinal Chemistry

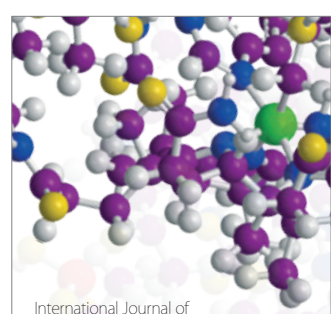

Carbohydrate Chemistry

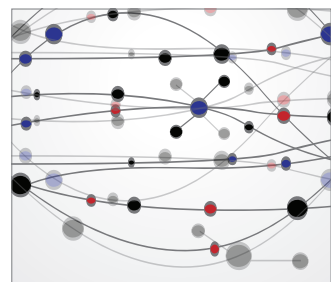

The Scientific World Journal
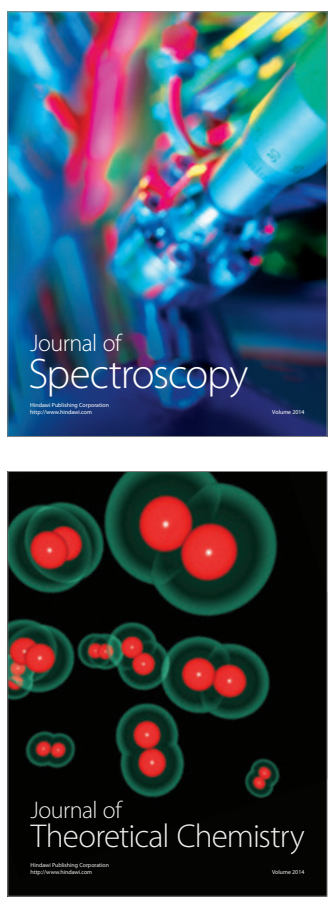
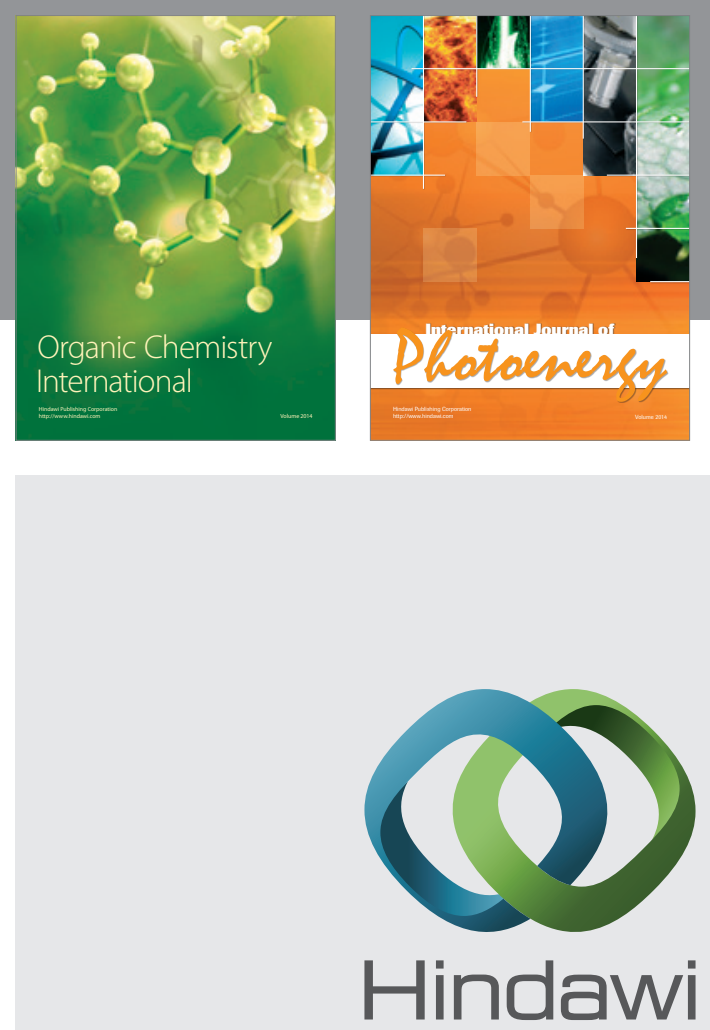

Submit your manuscripts at

http://www.hindawi.com
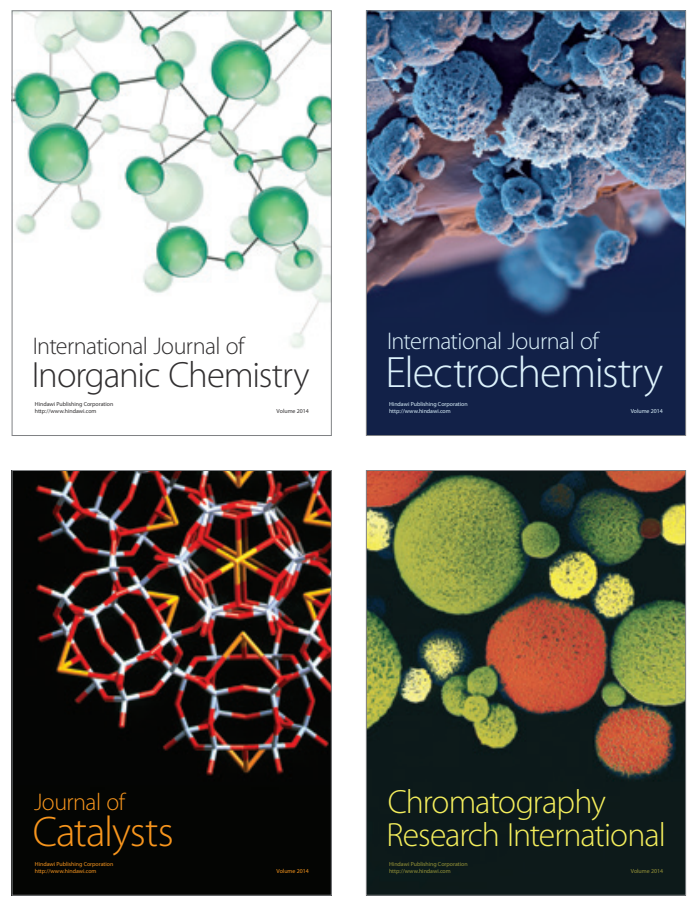
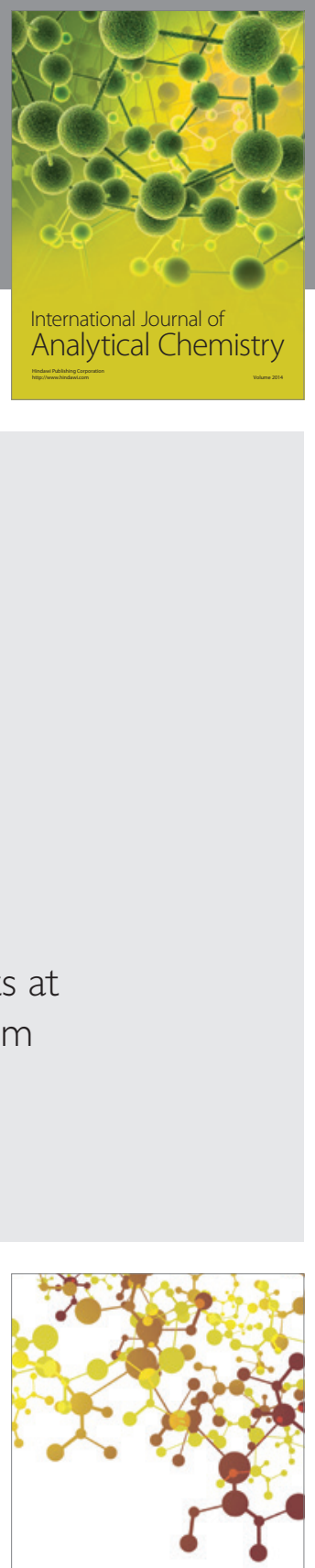

Journal of

Applied Chemistry
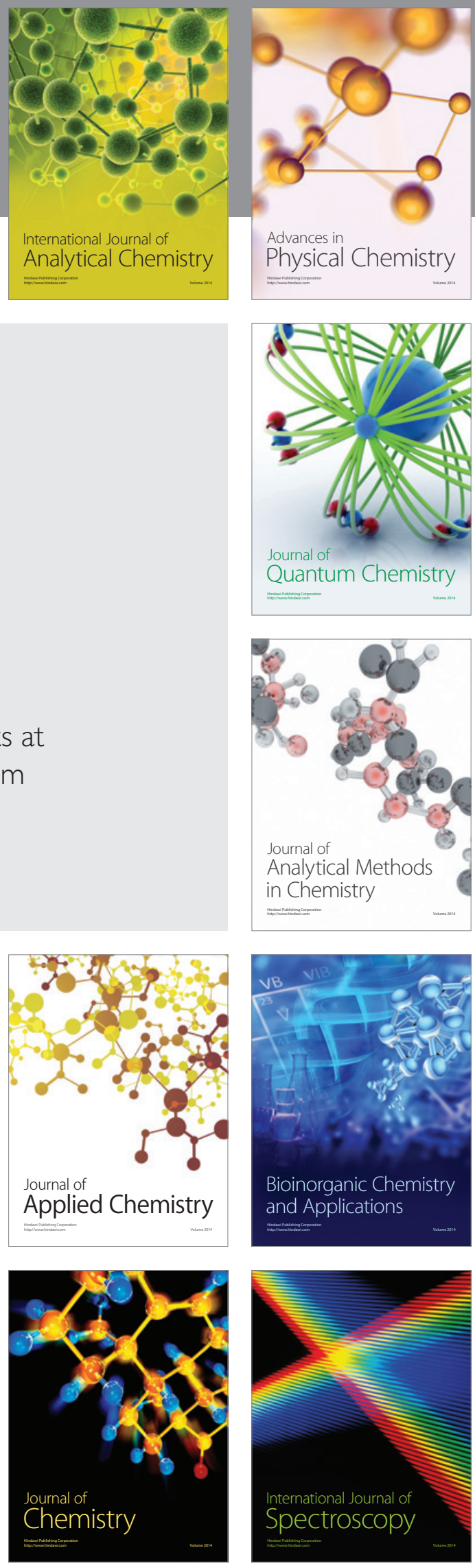